\title{
A Case of Cyclic Vomiting Syndrome Responding to Gonadotropin-Releasing Hormone Analogue
}

\author{
Young Kook Shin, M.D. ${ }^{1}$, Joong Goo Kwon, M.D. ${ }^{1 *}$, Ka Young Kim, M.D. ${ }^{1}$, Jae Bum Park, M.D. ${ }^{1}$, Seok Jae Han, M.D. ${ }^{1}$, Jong \\ Woon Cheon, M.D. ${ }^{1}$, Eun Young Kim, M.D. ${ }^{1}$, Ho Gak Kim, M.D. ${ }^{1}$, Tae Sung Lee, M.D. ${ }^{2}$, Kyung Sik Park, M.D. ${ }^{3}$, and Kyoung \\ Sook Won, M.D. ${ }^{4}$ \\ ${ }^{1}$ Departments of Internal Medicine and ${ }^{2}$ Obstetrics and Gynecology, Catholic University of Daegu School of Medicine, ${ }^{3}$ Departments of Internal \\ Medicine and ${ }^{4}$ Nuclear Medicine, Keimyung University Dongsan Medical Center, Daegu, Korea
}

Cyclic vomiting syndrome (CVS) is a disorder characterized by recurrent episodes of incapacitating nausea and vomiting interspersed with symptom free periods. Common triggers of cyclic vomiting include noxious stress, excitement, fatigue and menstrual period. Here, we report a case of cyclic vomiting syndrome in adult patient characterized by stereotypical vomiting attack, occurring in every menstruation period. Recurrent vomiting episodes began 6 years ago and we treated this patient with subcutaneous injection of goserelin, a gonadotropin-releasing hormone analogue ( $\mathrm{GnRHa}$ ) and oral estrogen. After 4 months of therapy, she was symptom free for the following 5 years, even with the resumed normal menstruation. Recurrence of vomiting attack with same pattern occurred 1 month before readmission. Treatment with intravenous lorazepam aborted vomiting, but could not prevent recurrences of vomiting and epigastric pain. We treated the patient with GnRHa and oral estradiol again which effectively prevented recurrence of the symptoms.

(J Neurogastroenterol Motil 2010;16:77-82)

Key Words

Cyclic vomiting syndrome, Menstrual period, Gonodotropin-releasing hormone analogue

\section{Introduction}

Cyclic vomiting syndrome (CVS) is a relatively rare disorder characterized by recurrent episodes of severe nausea and vomiting interspersed with symptom free periods. CVS remains as a clinical diagnosis which is based on three main criteria: stereotypical episodes of vomiting regarding acute onset and duration $(<1$ week), three or more discrete vomiting episodes in the pre- vious year and absence of nausea and vomiting between episodes. ${ }^{1-3}$ It was initially described in children, but could occur in any age groups. Recent studies have demonstrated that it is increasingly recognized as a cause of nausea and vomiting in adults and is highly disabling. ${ }^{4,5}$ CVS is a heterogeneous syndrome whose etiology is still unclear. Triggering factors of acute emetic episodes are similar in adults and children with CVS which include infections (e.g., chronic sinusitis, upper respiratory infections), emotional and psychological stress, motion sickness,

Received: December 3rd, 2009 Accepted: December 12th, 2009

(c) This is an Open Access article distributed under the terms of the Creative Commons Attribution Non-Commercial License (http://creativecommons. org/licenses/by-nc/3.0) which permits unrestricted non-commercial use, distribution, and reproduction in any medium, provided the original work is properly cited.

*Correspondence: Joong Goo Kwon, M.D.

Department of Internal Medicine, Catholic University of Daegu School of Medicine, 3056-6 Daemyung 4-dong, Nam-gu, Daegu 705-718, Korea

Financial support: None.

Tel: +82-53-650-4215, Fax: +82-53-628-4005, E-mail: kwonjg@cu.ac.kr

Conflicts of interest: None. 
lack of sleep, physical exhaustion and certain food products (e.g., chocolate, cheese, and monosodium glutamate). ${ }^{6}$ In some women, episodes are triggered by menstruation in which oral contraceptive pill could help to prevent symptoms. ${ }^{1,6}$ Here we report a case of adult woman who had menstruation related cyclic vomiting in whom GnRHa successfully prevented recurrence of the symptoms.

\section{Case Report}

A 23-year-old woman visited our emergency department for recurrent vomiting and epigastric pain that had developed 2 days ago. Six years ago, she experienced acute onset of vomiting and severe epigastric pain for the first time. After then, she had suffered from recurrent, stereotypic episodes of incapacitating nausea and vomiting, separated by intervals of symptom-free periods. Her symptoms occurred with the onset of menstrual period and lasted for 7-10 days. Recurrent vomiting, usually beginning in the late night, occurred 30-50 episodes per day and was usually associated with severe epigastric pain. After the end of menstrual period, the symptoms resolved spontaneously. Past medical and surgical history were unremarkable. The age of menarche was 13. Duration of menstrual flow was 7-10 days and amount of menstrual flow was medium. Menstrual cycle was regular and mean duration was 30 days. She had not taken any medication. Her younger sister suffered from migraine with aura (Fig. 1). Physical examination, laboratory test, endoscopic examination and evaluation by gynecologist revealed no causative explanation for her symptoms. Management with proton pump inhibitor, analgesics, antiemetics or prokinetics did not affect the duration and severity of symptom episode. For management of dysmenorrhea, two monthly cycles of estrogen-progestin hormonal contraceptives were administered, but it did not prevent recurrence of the symptoms. In order to induce amenorrhea, we treated her with subcutaneous injection of $\mathrm{GnRHa}$ (goserelin, $3.6 \mathrm{mg} / \mathrm{month}$ ) and oral estrogen (premarin ${ }^{\circledR}, 0.625 \mathrm{mg} /$ day) for 4 months. After then, menstruation disappeared for 6 months and she had experienced 5 years of symptom-free period, even with the resumed normal menstruation. For the past 3 months before readmission, she had experienced anxiety and stress due to an examination. Recurrence of vomiting attack with same pattern occurred 1 month before readmission which required hospitalization for intravenous hydration and symptom reduction. Treatment with intravenous lorazepam aborted symptoms of emetic phase. But, trials of fluoxetine, a selective serotonin reuptake inhibitors (SSRI),

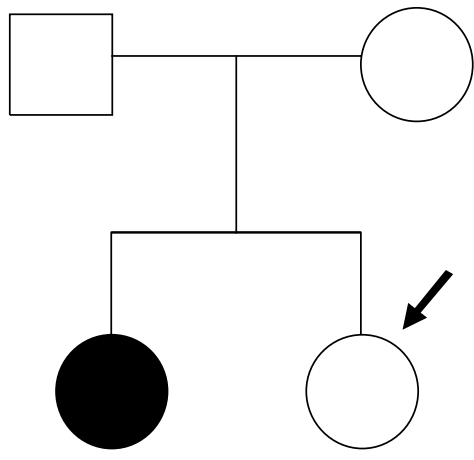

Figure 1. Pedigree of the patient's family. In this pedigree, one patient with cyclic vomiting syndrome (solid circle) and her younger sister who had migraine headache (arrow) were detected.

could not prevent recurrence of the symptoms on the next menstrual period. Upon admission, she had frequent nausea, vomiting and severe epigastric pain, especially at late night and early morning. On physical examination, patient's vital signs were stable and significant orthostatic change was not seen. The abdomen was soft, non-distended with normal bowel sound, but epigastric tenderness was noted. Neurological findings were unremarkable. Laboratory evaluation revealed white bood cell of $8,900 / \mathrm{mm}^{3}$, hemoglobin $12.8 \mathrm{~g} / \mathrm{dL}$, platelet $458,000 / \mathrm{mm}^{3}$, serum sodium 139 $\mathrm{mEq} / \mathrm{L}$, serum potassium $3.9 \mathrm{mEq} / \mathrm{L}$, BUN $20 \mathrm{mg} / \mathrm{dL}$, serum creatinine $0.8 \mathrm{mg} / \mathrm{dL}$, aspartate aminotransferase $16 \mathrm{IU} / \mathrm{L}$, alanine aminotransferase $28 \mathrm{IU} / \mathrm{L}$, total protein $8.1 \mathrm{~g} / \mathrm{dL}$, albumin $4.7 \mathrm{~g} / \mathrm{dL}$, TSH $2.34 \mathrm{IU} / \mathrm{mL}$, and free T4 1.21 IU/mL. Urinary pregnancy testing was negative. An extensive gastrointestinal workup including upper gastrointestinal endoscopy, upper gastrointestinal series with barium, abdomino-pelvic CT scans, electrogastrogram (EGG) and gastric emptying scintiscans with ${ }^{99 \mathrm{~m}}$ Tc-DTPA were done. Some of these studies were performed while the patient was symptomatic. EGG and gastric emptying scintiscans were performed just after improvement of symptoms and discontinuation of any prokinetic agent. Upper GI endoscopy showed tongue-like mucosal projection just above the esophagogastric junction (Fig. 2). Upper GI series and abdomino-pelvic CT scans identified no abnormality. EGG showed normally increased total power at post-prandial period and no bradygastria or tachygastria was identified (Fig. 3). Gastric emptying scintiscans demonstrated delayed gastric emptying. Half emptying time $\left(T_{1 / 2}\right)$ was 107 minutes and percentage retained at 4 hours was $22 \%$ (Fig. 4). Management with intravenous hydration, antiemetics and lorazepam reduced severity and duration of the symptoms. After then, we treated her with subcutaneous in- 


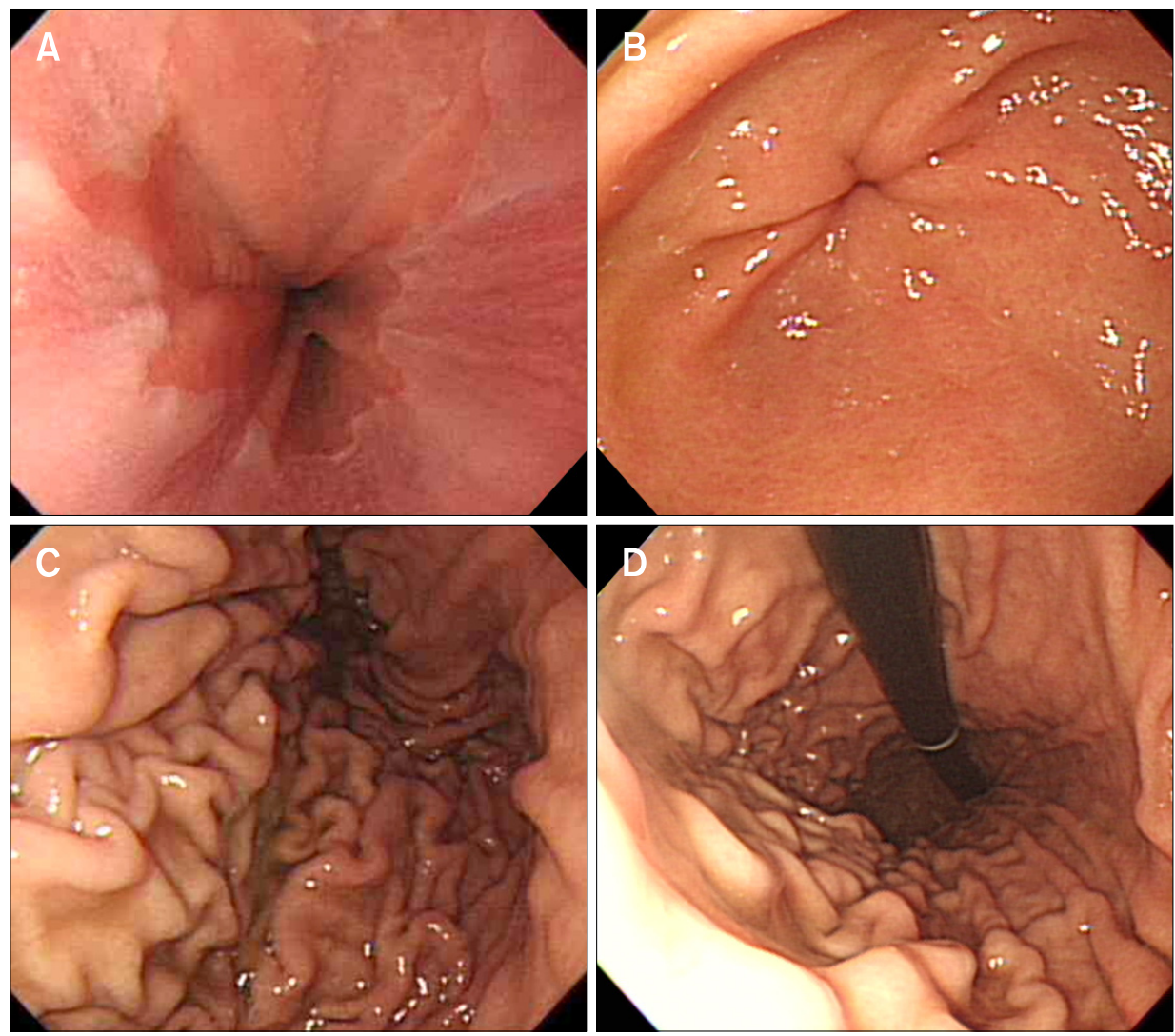

Figure 2. Upper GI endoscopic findings showing tongue-like mucosal projection just above the esophagogastric junction (A). Neither mucosal abnormality nor luminal narrowing is identified in the stomach (B-D).

jections of GnRHa (goserelin $3.6 \mathrm{mg} / \mathrm{month}$ or leuprolide 3.75 $\mathrm{mg} / \mathrm{month}$ ) and oral estradiol ( $2 \mathrm{mg} /$ day) for 3 months and the patient showed no recurrence of the symptoms for 6 months during follow-up visits.

\section{Discussion}

CVS is a rare disorder diagnosed primarily in children and frequently misdiagnosed as acute gastroenteritis, gastroesophageal reflux disease, pancreatitis or eating disorders. ${ }^{2,7,8}$ Patients with CVS typically present with recurrent stereotypic episodes of nausea and vomiting lasting hours or days and each episode is separated by symptom free-intervals, which last weeks or months. Vomiting typically begins during the night or early morning and peaks in the first hour, occurring up to 13 times per hour. ${ }^{3}$

Classically perceived as a pediatric disorder, CVS is being recognized increasingly in adults. Cyclic vomiting episodes in children cease spontaneously before puberty in most cases, but persistence through to adult life is well recognized. Adults with CVS can occur at any age, with an average age of onset of 21-35 years in limited case series. Although the clinical features in each age groups are similar, ${ }^{9}$ a few differences in symptom profile, pathogenesis and associated comorbidities between adults and children have been observed. ${ }^{1}$ Median interval of episode is 4 weeks in children and 3 months in adults. ${ }^{10}$ Nausea or dyspepsia between episodes is more common in adults. Delays in diagnosis appear less frequently in pediatric patients (1.9 years) than in adults (7.9 years). ${ }^{11}$

The pathophysiology of CVS has not been established, and recent investigations suggest that several factors, including metabolic disorders, migraines, autonomic dysregulation, hypothalamic-pituitary-adrenal axis defects, a tendency for anxiety and chronic cannabis use could precipitate CVS in some cases. 68-80\% of CVS attacks have associated trigger mechanisms such as infection, psychological stress, physical stress, inadequate sleep, diet, motion sickness and onset of menses. ${ }^{6} \mathrm{Up}$ to $13 \%$ of postmenarchial girls develop CVS at the onset of menstrual period (named catamenial CVS). ${ }^{12}$ We suggest that anticipatory anxiety and stress for an examination and menstruation together contributed to the triggering factors of vomiting attacks in our case. 5 years of symptom-free period may be related with period of reduced anxiety and stress. 

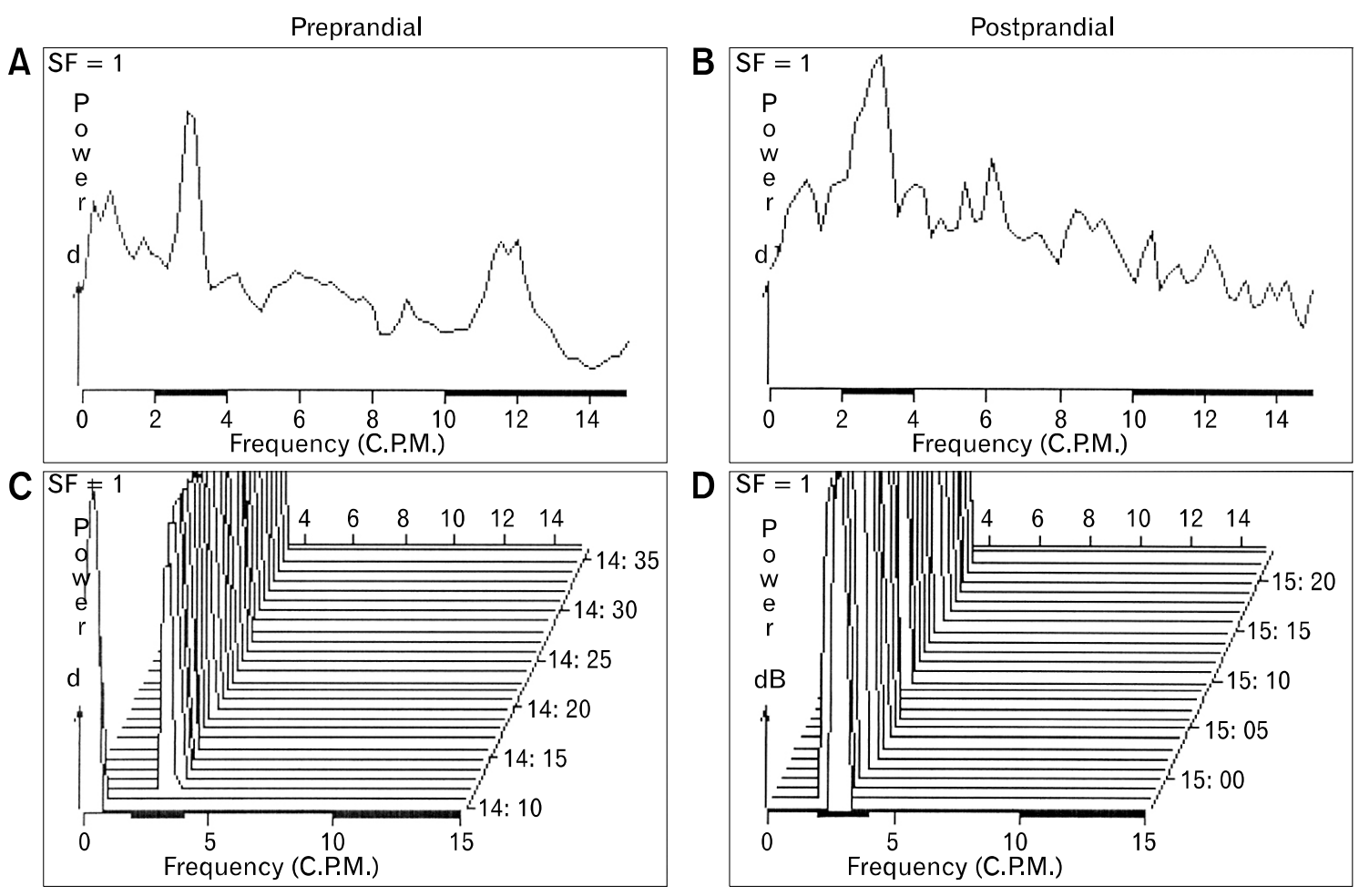

Figure 3. Electrogastrogram findings showing normally increased total power at postprandial period (A, B). Neither bradygastria nor tachygastria is identified (C, D).

CVS remains a diagnosis based upon the history and exclusion of alternative diagnoses. Rome III criteria include the stereotypical episodes of vomiting, three or more discrete episodes, and symptom free periods. The criteria should be fulfilled for the last three months with symptom onset at least six months before diagnosis. Supportive criteria includes history or family history of migraine headaches. ${ }^{3}$ The recurrent vomiting attacks and symptom-free interval of our case met the consensus criteria of CVS and family history revealed migraine headache of the sister. Duration of episode in our case is not consistent with Rome III criteria. However, longer duration of episodes ( $>1$ week) has been found in some adult patients with cyclic vomiting syndrome. $^{12}$

Differential diagnosis of CVS is very broad and it is important to exclude other gastrointestinal disorders and extra-intestinal disorders, including metabolic and central nervous system diseases, renal disorders, and psychogenic vomiting. The extent of diagnostic evaluation and the role for the use of empiric therapy for migraine in patients with CVS remains undefined. Diagnostic approach to exclude other disorders with recurrent vomiting includes biochemical testing for electrolyte abnormalities, serum calcium, thyroid function tests and liver function tests. An upper GI series with small bowel follow-through X-ray, $\mathrm{CT} / \mathrm{MRI}$ of the head, and endoscopy can be performed in between episodes. ${ }^{7,8,13}$ Because the vomiting attacks only occurred at the onset of menstruation, we suspected dysmenorrhea as a cause of symptoms. But, neither the typical manifestations of dysmenorrhea were identified nor the treatment for it controlled the symptoms.

Gastric emptying scintiscans have been investigated in adult patients with CVS, primarily during asymptomatic periods in between emetic episodes. Rapid gastric emptying at baseline, which may be related to autonomic dysfunction, or delayed gastric emptying during episodes have been demonstrated in adults with CVS. ${ }^{14,15}$ In our case, gastroparesis was initially considered as a cause of frequent vomiting episodes. But, trials with prokinetics including erythromycin, mosapride and antiemetics did not affect the duration and severity of symptom episode. Moreover, on-off symptom pattern with intervals of complete normality in between menstruation suggests uncertain association between vomiting episodes and gastroparesis.

No specific therapy has been proven to be effective for CVS in controlled trials. However, several empiric treatments have been effective in case series. ${ }^{16}$ Life style changes, abortive treat- 


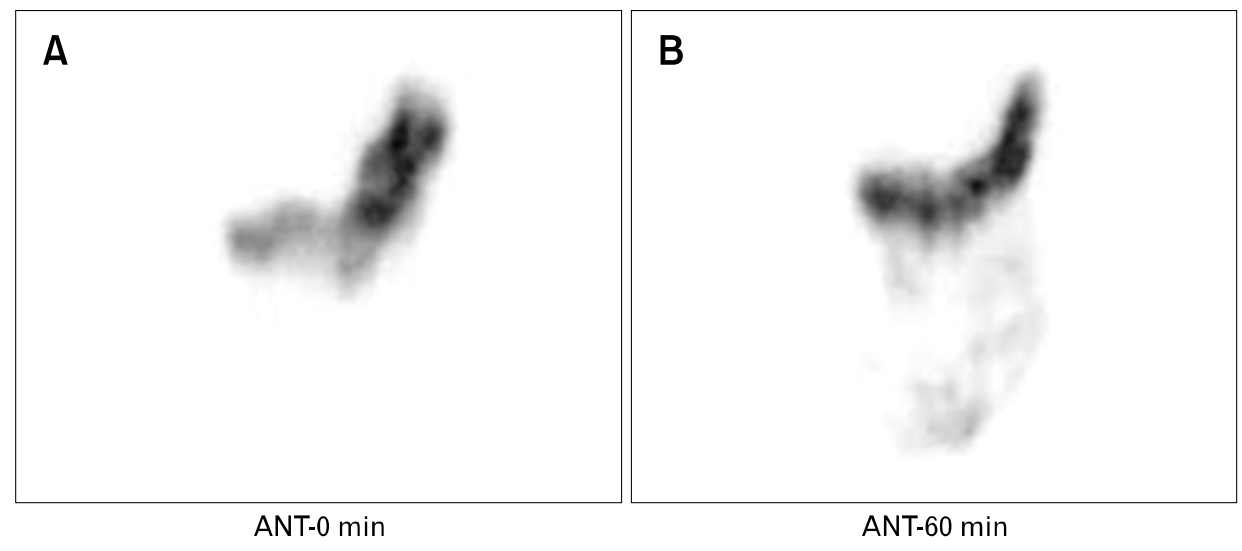

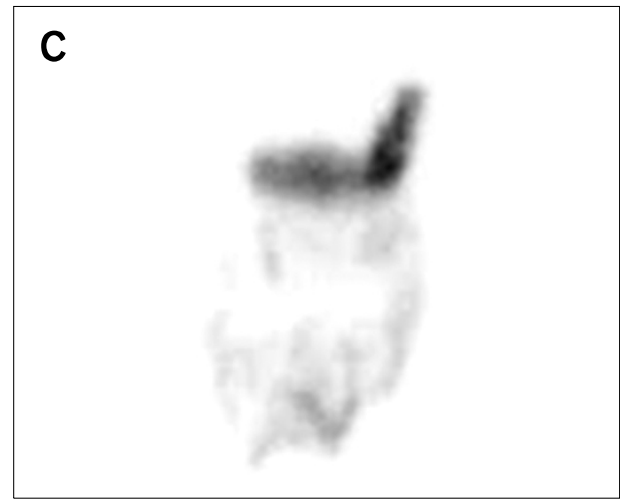

ANT-120 min

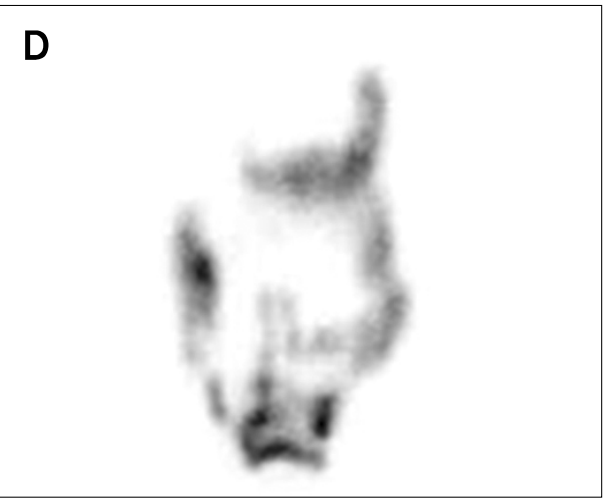

ANT-240 $\min$
Figure 4. Gastric emptying scintiscans showing delayed gastric emptying (A-D). Half emptying time $(\mathrm{T} 1 / 2)$ is $107 \mathrm{mi}$ nutes and the percentage retained at 4 hour is $22 \%$ (D). ment, prophylactic therapy, and supportive care to ameliorate acute episodes are efficient treatments. Avoidance of potential triggers is helpful. Diabetes should be managed with strict glucose control and episodes of hypoglycemia should be avoided. Treatments during the emetic phase consist of hydration, antiemetic, antianxiety and/or analgesic medications. Supportive care with fluid replacement and electrolyte control is often necessary. A constant intravenous infusion of $5-\mathrm{HT}_{3}$ antagonist, ondansetron, has shown an efficacy of about $60 \%$ if given early in the vomiting attack. ${ }^{17}$ If nausea persists, addition of lorazepam provides sedation that may lessen intractable nausea in some cases. ${ }^{11}$ In our case, repeated injections of lorazepam during the emetic phase reduced both nausea and vomiting. But, treatment with lorazepam and fluoxetine did not prevent recurrence of symptoms.

Some studies have investigated the drug therapy in preventing episodes of CVS and only a small number of maintenance therapies have so far been reported to be effective. Low-dose tricyclic antidepressants, sumatriptan, and beta blockers (e.g., propranolol) have been found to be effective in prevention of episodes. ${ }^{1,18}$ If psychiatric disorder is present, it should also be treated. If menstrual cycles trigger an attack, GnRHa injections or continuous birth control to block menses should be considered. Although low-dose estrogen oral contraceptives could help to prevent the vomiting attack in patients with menstruationrelated CVS, ${ }^{11}$ oral contraceptives can also exacerbate symptoms on the other hand. ${ }^{16,19}$ Administration of GnRHa down-regulates the pituitary-ovarian gonadal axis and reduces levels of the gonadotropin, luteinizing hormone and follicle-stimulating hormone. ${ }^{20}$ GnRHa suppresses ovulation and is found to have benefits in the treatment of premenstrual syndrome. However, maximum treatment period is 6 months, and hormone replacement therapy has to be given to prevent side effects. ${ }^{21}$ We chose GnRHa and estrogen replacement therapy as the prophylactic therapeutic modality due to its efficacy in preventing vomiting attack and a relatively short period of courses of therapy (e.g., 3 months).

In conclusion, careful understanding of the history, symptom pattern and triggering factors should be obtained in patients with recurrent vomiting attacks. For women with menstruation-related CVS, we suggest that GnRHa is an effective therapeutic modality for preventing symptom episode. 


\section{References}

1. Abell TL, Adams KA, Boles RG. Cyclic vomiting syndrome in adults. Neurogastroenterol Motil 2008;20:269-284.

2. Li BU, Balint JP. Cyclic vomiting syndrome: Evolution in understanding of a brain-gut disorder. Adv Pediatr 2000;47:117-160.

3. Tack J, Talley NJ, Camilleri M, et al. Functional gastroduodenal disorders. Gastroenterology 2006;130:1466-1479.

4. Fitzpatrick E, Bourke B, Drumm B, Rowland M. The incidence of cyclic vomiting syndrome in children: population-based study. Am J Gastroenterol 2008;103:991-995.

5. Li BU, Misiewicz L. Cyclic vomiting syndrome: a brain-gut disorder. Gastroenterol Clin North Am 2003;32:997-1019.

6. Pareek N, Fleisher DR, Abell T. Cyclic vomiting syndrome: what a gastroenterologist needs to know. Am J Gastroenterol 2007;102: 2832-2840.

7. Forbes D. Differential diagnosis of cyclic vomiting syndrome. J Pediatr Gastroenterol Nutr 1995;21(supple 1):S11-S14.

8. Li BU. Cyclic vomiting syndrome: Age-Old syndrome and new insights. Semin Pediatr Neurol 2001;8:13-21.

9. Prakash C, Staiano A, Rothbaum RJ, Clouse RE. Similarities in cyclic vomiting syndrome across age groups. Am J Gastroenterol 2001;96:684-688.

10. Fleisher DR, Matar M. The cyclic vomiting syndrome: a report of 71 cases and literature review. J Pediatr Gastroenterol Nutr 1993; 17:361-369.

11. Sunku B. Cyclic vomiting syndrome: a disorder of all ages. Gastroenterol Hepatol 2009;5:507-515.
12. Fleisher DR, Gornowicz B, Adams K, Burch R, Feldman EJ. Cyclic vomiting syndrome in 41 adults: the illness, the patients and the problems of management. BMC Med 2005;3:20-31.

13. Catto-Smith AG, Ranuh R. Abdominal migraine and cyclic vomiting. Semin Pediatr Surg 2003;12:254-258.

14. Namin F, Patel J, Lin Z, et al. Clinical, psychiatric and manometric profile of cyclic vomiting syndrome in adults and response to tricyclic therapy. Neurogastroenterol Motil 2007;19:190-202.

15. Fajardo NR, Locke GR, Talley NJ. Cyclic vomiting syndrome is associated with rapid early gastric emptying. Am J Gastroenterol 2005;100:S70.

16. Li BU, Lefevre F, Chelimsky GG, et al. North American Society for Pediatric Gastroenterology, Hepatology, and Nutrition consensus statement on the diagnosis and management of cyclic vomiting syndrome. J Pediatr Gastroenterol Nutr 2008;47:379-393.

17. Li BU, Murray RD, Heitlinger LA, Robbins JL, Hayes JR. Is cyclic vomiting syndrome related to migraine? J Pediatr 1999;134:567572.

18. Benson JM, Zorn SL, Book LS. Sumatriptan in the treatment of cyclic vomiting. Ann Pharmacother 1995;29:997-999.

19. Boyle CA. Management of menstrual migraine. Neurology 1999; 53(supple 1):S14-S18.

20. Lyall H, Campbell-Brown M, Walker JJ. GnRH analogue in everyday gynecology: is it possible to rationalize its use? Acta Obstet Gynecol Scand 1999;78:340-345.

21. West CP, Hiller H. Ovarian suppression with the gonadotrophin-releasing hormone agonist goserelin (Zoladex) in the management of the premenstrual tension syndrome. Human reproduction 1994;9:1058-1063. 